\title{
Datos microtermométricos de algunas unidades litológicas del subterreno Teloloapan, Guerrero, México
}

\author{
Eduardo González-Partida ${ }^{1 *}$, Juan Carlos Salinas-Prieto ${ }^{2}$ y Martha Moctezuma-Salgado ${ }^{2}$ \\ ${ }^{1}$ Unidad de Investigación en Ciencias de la Tierra, Instituto de Geología, Universidad Nacional \\ Autónoma de México, Campus UNAM - Juriquilla, A.P. 1-742, Querétaro, Qro. C.P. 76230 \\ 2 Consejo de Recursos Minerales, Blv. Felipe Angeles S/N, Carr. México-Pachuca Km 93-5, \\ Pachuca Hgo., CP 42080 \\ * egp@unicit.unam.mx
}

\section{Resumen}

Las inclusiones fluidas estudiadas en filitas y metavolcanoclásticos en Taxco y Taxco el Viejo son del tipo líquido+vapor $(\mathrm{L}+\mathrm{V})$ a líquido dominante, con presencia de gases carbónicos y con temperaturas de homogeneización (Th) de $205^{\circ} \mathrm{a} 250^{\circ} \mathrm{C}$. A baja temperatura los fluidos son de dos tipos: 1) del sistema $\mathrm{H}_{2} \mathrm{O}-\mathrm{NaCl}$ y 2) fluidos con $\mathrm{H}_{2} \mathrm{O}+$ sales+compuestos carbónicos. Los del primer tipo presentan temperaturas de fusión (Tf) de $-7.6^{\circ} \mathrm{a}-19.8^{\circ} \mathrm{C}$, lo que implica salinidades del orden de 11.22 a $22.24 \%$ eq. peso de $\mathrm{NaCl}$. En los metavolcanoclásticos de Teloloapan, los fluidos son a $\mathrm{L}+\mathrm{V}$. Presentan $\mathrm{Th}=141^{\circ}$ a $193^{\circ} \mathrm{C}$ y $\mathrm{Tf}=-1.5^{\circ}$ a $-6.4^{\circ} \mathrm{C}$, lo que corresponde a salinidades de 2.5 a $9.7 \%$ eq. peso de $\mathrm{NaCl}$. En la Formación Amatepec la temperatura de homogeneización promedio fue de $132{ }^{\circ} \mathrm{C}$ con presencia de $\mathrm{CO}_{2}$ en clataratos y abundancia de inclusiones fluidas unifásicas de líquido.

Estos datos microtérmicos representan los valores mínimos del evento metamórfico y muestran una diferencia significativa con los esquistos negros de Tejupilco estudiados con anterioridad. Éstos, por más de veinte años se han agrupado con los metavolcanoclásticos de Teloloapan y los esquistos de Taxco y Taxco el Viejo, lo que ha conducido a atribuir edades muy diferentes a todos estos conjuntos (desde Paleozoico hasta Cretácico). En el presente trabajo se presume que los esquistos negros de Tejupilco (prejurásicos) presentan una historia estructural y térmica diferente a la observada en los metavolcanoclásticos que afloran en Teloloapan y Taxco y en la Formación Amatepec, miembros del subterreno Teloloapan del Jurásico-Cretácico.

Palabras clave: inclusiones fluidas, microtermometría, Formación Amatepec, subterreno Teloloapan, Guerrero, México

\begin{abstract}
The fluid inclusions studied in slates and metavolcanoclastic rocks in Taxco and Taxco El Viejo are liquid dominant liquid + vapor $(\mathrm{L}+\mathrm{V})$ with some carbonic gas $\left(\mathrm{CO}_{2}\right)$. The homogenization temperatures $(\mathrm{Th})$ values range between $205^{\circ}$ and $250^{\circ} \mathrm{C}$. At low temperature, the fluids are of two types: 1) $\mathrm{H}_{2} \mathrm{O}-\mathrm{NaCl}$; and 2) $\mathrm{H}_{2} \mathrm{O}+$ salts+organic compounds. The first type of fluids presents melting temperatures (Tf) ranging between $-7.6^{\circ}$ to $-19.8^{\circ} \mathrm{C}$. This implies salinities between 11.22 to $22.4 \%$ wt. eq. $\mathrm{NaCl}$. Metavolcanoclastic rocks in Teloloapan contain inclusions that are L+V. These inclusions present $\mathrm{Th}=141$ to $193^{\circ} \mathrm{C}$ and $\mathrm{Tf}=-1.5$ to $-6.4^{\circ} \mathrm{C}$, corresponding to salinities between 2.5 and $9.7 \%$ wt. eq. $\mathrm{NaCl}$. The average Th of inclusions within the Amatepec Formation is $132^{\circ} \mathrm{C}$ with the presence of $\mathrm{CO}_{2}$ in clatherates and abundant liquid uniphasic fluid inclusions.

These microthermic data represent the minimum values for the metamorphic event and show a significant difference with the previously studied Black Schist of Tejupilco. For over twenty years, these Black Schist have been grouped with the metavolcanoclastics rocks of Teloloapan and the Taxco and Taxco El Viejo Schist, which have lead to assign different ages to these rocks (from Paleozoic to Cretaceous). In this paper, we conclude that the Black Schist of Tejupilco (pre-Jurassic) shows a structural and thermal history different to the metavolcanoclastics rocks of the Teloloapan and Taxco, and to the Amatepec Formation of the Jurassic-Cretaceous Teloloapan sub-terrain.
\end{abstract}

Keywords: fluid inclusions, microthermometry, Formación Amatepec, Teloloapan sub-terrain, Guerrero, Mexico 


\section{Introducción}

Por tres décadas ha existido fuerte controversia sobre las características cronológicas y petrológicas de algunas unidades litológicas de Tierra Caliente. Esta controversia surgió a raíz del trabajo de De Cserna et al. (1974), cuestionado por Campa et al. (1974) y Campa (1978), lo cual motivó el desarrollo de tesis de maestría y doctorado, así como trabajos de investigación enfocados a aspectos petrológicos y estructurales. Entre estos trabajos destacan los de Colorado-Liévano (1979), Ortiz-Hernández et al. (1991), Ortiz-Hernández (1992), Tolson (1993), Talavera-Mendoza (1993), Talavera-Mendoza et al. (1995) y Salinas-Prieto (1994), quienes aportaron datos sobre el comportamiento tectónico y petrológico de estas unidades litológicas. Ninguno de estos autores estudió el comportamiento de los fluidos relacionados con los procesos diagenéticos y metamórficos.

Estudios de inclusiones fluidas fueron practicados por González-Partida (1993a) en los Esquistos Negros de Tejupilco, presumiblemente prejurásicos pero considerados por de Cserna y Fries (1981) como del Paleozoico. Esta clase de estudios son los únicos que se han realizado en el país en rocas metamórficas y, para complementar esta información, en este trabajo se presentan datos microtermométricos a partir del estudio de inclusiones fluidas de 17 muestras de cuarzo y calcita de segregación tomadas de los denominados Esquistos Taxco (de Cserna 1982), de las rocas metavolcanoclásticas de Teloloapan y de la Formación Amatepec, miembros todos del subterreno Teloloapan (Salinas-Prieto 1994), y que sobreyacen a los Esquistos Negros de Tejupilco.

\section{Contexto geológico}

La porción norte del Estado de Guerrero, en su límite con el Estado de México, se caracteriza por presentar afloramientos de rocas metamórficas de bajo grado pertenecientes a secuencias metavolcanoclásticas de arco. Estas rocas, como ya se mencionó, han sido estudiadas desde diferentes puntos de vista, sin embargo, existe aún controversia sobre las diferentes unidades tectónicas que se presentan en la zona.

En la región señalada en la Figura 1 afloran rocas del Terreno Guerrero y del Terreno Mixteco (Campa y Coney 1983) en contacto tectónico a través de cabalgaduras. El primero está representado en esta región por los llamados subterrenos Arcelia (STA) y Teloloapan (STT). Del Terreno Mixteco aflora únicamente la Plataforma carbonatada Guerrero-Morelos.

El STA está caracterizado por la presencia de coladas de lavas basálticas almohadilladas cortadas por filones doleríticos (Ortiz-Hernández 1992; Talavera-Mendoza 1993). Las lavas se intercalan con capas ricas en radiolarios. Hacia la cima, las intercalaciones de lava con sedimentos silíceos radiolaríticos son más frecuentes e inicia una interestratificación con tobas y arenas volcánicas.
Un flysch predominantemente arenoso que contiene bloques de rocas básicas, brechas magmáticas y calizas, corona el conjunto metavolcanoclástico en continuidad. Diversos fechamientos isotópicos (Delgado-Argote et al. 1990; Elías-Herrera y Sánchez-Zavala 1990) ubican a las lavas en el nivel del Aptiano al Cenomaniano, mientras que las determinaciones paleontológicas en radiolarios de la secuencia confirman edades del Cretácico Inferior (Salinas-Prieto 1994).

El vulcanismo basáltico submarino del STA muestra afinidad con toleitas de arco (Ortiz-Hernández et al. 1991; Talavera-Mendoza 1993) y representaría parte del testigo de un arco insular intraoceánico (TalaveraMendoza 1993). El STA está afectado por un metamorfismo de facies prehnita-pumpelita (Talavera-Mendoza 1993). Los sedimentos asociados al conjunto metavolcanoclástico muestran con mucha frecuencia una estructura penetrativa característica de una deformación no-coaxial de baja temperatura, con cinemática que indica movimiento de la parte superior hacia el Este (Salinas-Prieto 1994).

El STT está constituido típicamente por dos conjuntos litológicos: rocas metavolcanoclásticas pertenecientes a un arco de edad Jurásico Superior-Cretácico Inferior, y una cubierta sedimentaria del Cretácico InferiorCretácico Superior, donde se encuentra la Formación Amatepec. El primero representa una sucesión volcánica del arco propiamente dicho. Está compuesto de alternancias de basaltos en coladas y almohadillas, de autobrechas de almohadillas y de arenas volcánicas, cubiertas en concordancia por sedimentos tobáceos y radiolaríticos. Hacia la cima, los niveles sedimentarios devienen más abundantes y se interestratifican con tobas y conglomerados volcánicos. Las coladas de lava coexisten con calizas bioclásticas. Talavera-Mendoza et al. (1990, 1992), Talavera-Mendoza (1993) y González-Partida (1993b) proponen que las lavas son típicas de series de arco calcialcalino evolucionado, con una componente inicial alcalina. La cubierta sedimentaria, que reposa en transición sobre el arco, está constituida por sedimentos volcanodetríticos interestratificados con lentes de caliza del Aptiano Superior (Campa et al. 1974). Estos lentes de caliza pasan progresivamente a niveles calcáreos subarrecifales y detríticos con material volcánico retrabajado en la base. En la región occidental, la secuencia carbonatada está representada por calcarenitas negras con niveles pelíticos. La edad de las calizas ha sido determinada por la presencia de fauna fósil de edad Aptiano superiorAlbiano superior (Monod y Busnardo 1992). Las calizas están cubiertas en forma transicional por sedimentos areno-arcillosos tipo flysch con edad mínima del Cenomaniano (Monod y Busnardo 1992).

La Formación Amatepec fue definida por De Cserna (1982) en el poblado del mismo nombre y corresponde a rocas arcillosas-carbonatadas, predominando los carbonatos de horizontes delgados.

El conjunto del STT está afectado por metamorfismo de facies de esquistos verdes ligado al desarro- 


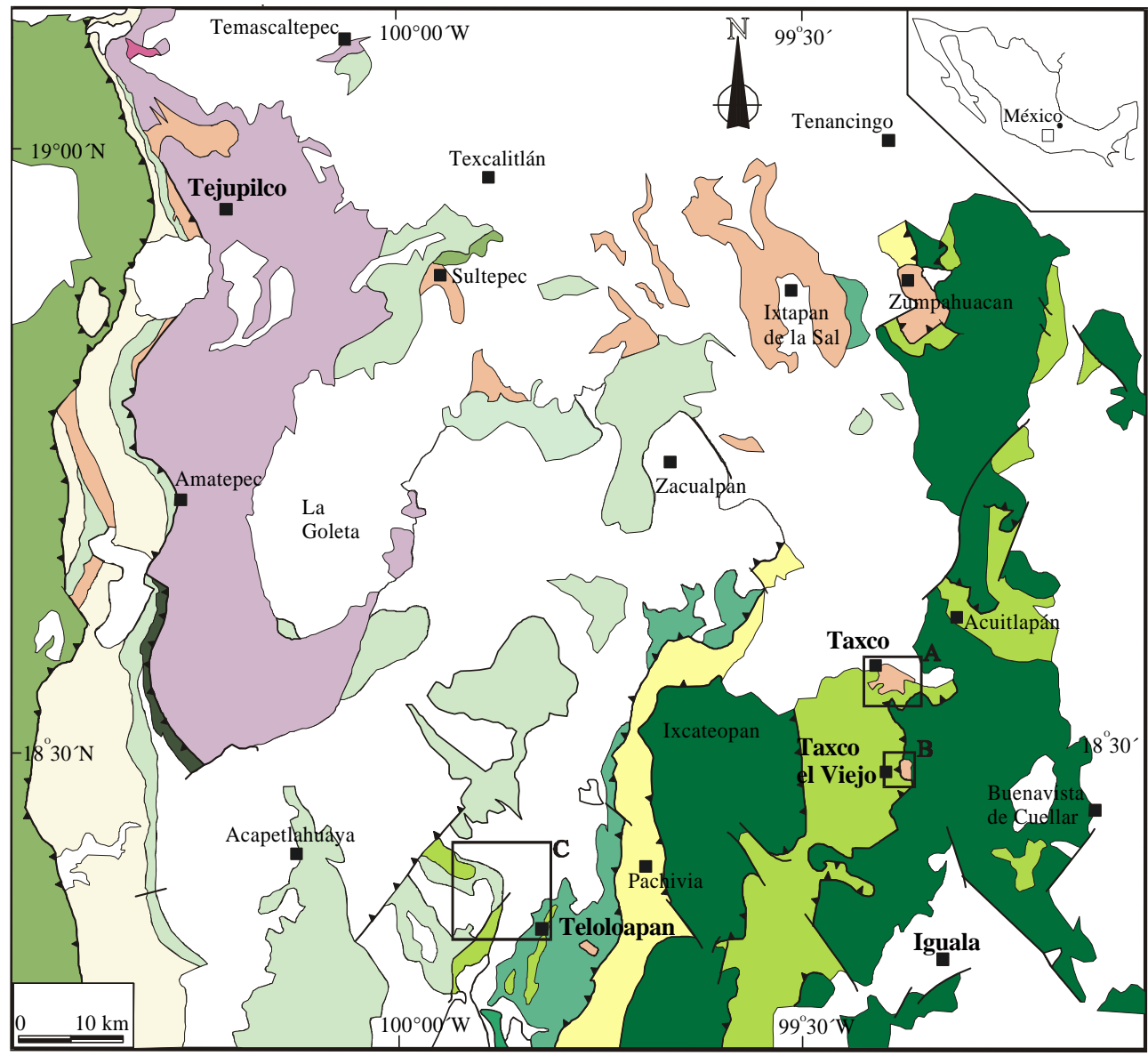

\section{LEYENDA}

Terciario y Cuaternario

$\square$ Formaciones post-tectónicas

Conglomerado Balsas

Plataforma Morelos-Guerrero

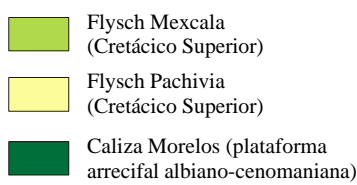

Subterreno Teloloapan

$\square$ Flysch de Miahuatepec Cretácico)

Calizas bioclásticas de

Teloloapan (Aptiano-Albiano)

Calizas micríticas negras

(Fm. Amatepec)

Lavas y depósitos

volcaniclásticos de arco

(Jurásico Sup.-Cretáico Inf.)

Areniscas y tobas basales

Subterreno Arcelia

$\square$ Lavas almohadilladas, pedernal y areniscas (Cretácico Inf.)

\section{Basamento}

Esquistos de Tejupilco

(prejurásico)

Ortogneis de Tizapa (prejurásico)

- Sitios de muestreo
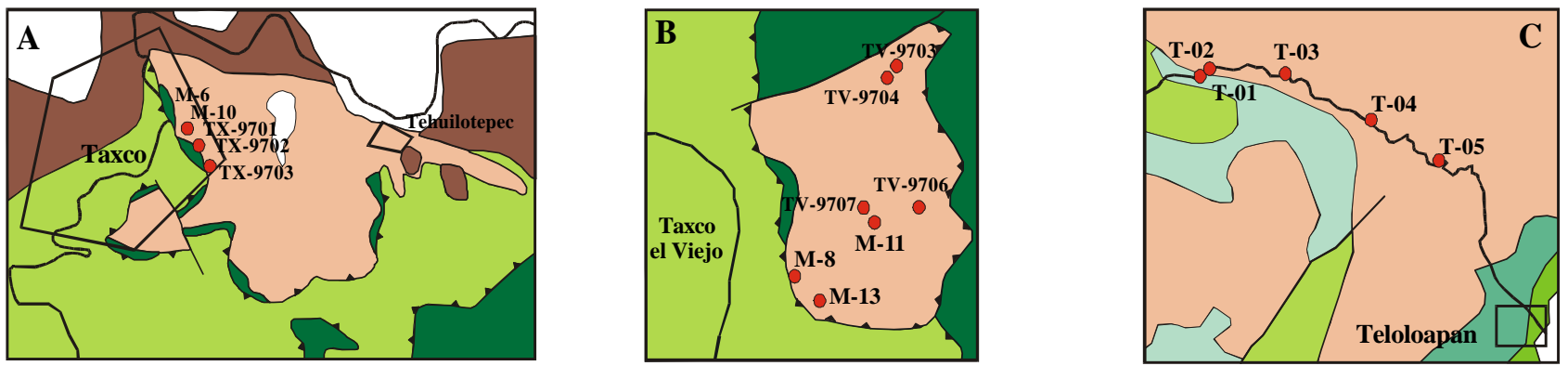

Figura 1. Carta geológica del Subterreno Teloloapan (Modificado de Salinas-Prieto, 1994)

llo de esquistocidad, cuyas condiciones dinámicas fueron provocadas por la tectonización del subterreno en condiciones dúctiles de cizalla no-coaxial de baja temperatura (Salinas-Prieto 1994).

La Plataforma Guerrero-Morelos, que pertenece a la cubierta sedimentaria del Terreno Mixteco, consiste en calizas arrecifales y subarrecifales del AlbianoCenomaniano cubiertas por sedimentos tipo flysch del Cretácico Superior (Formaciones Morelos y Mexcala). Este conjunto presenta desarrollo de superficies de esquistocidad sólo en las porciones cercanas a las estruc- turas mayores de cabalgadura con orientación sensiblemente norte-sur.

Las condiciones, estilos y cinemática de deformación dúctil son similares tanto en los subterrenos Arcelia y Teloloapan como en la Plataforma Guerrero-Morelos (Salinas-Prieto 1994).

Dos regiones dentro del área trabajada presentan características particulares que requieren de un tratamiento aparte: la región de Tejupilco y la de TaxcoTaxco el Viejo. La primera es una región donde afloran esquistos cuarzofeldespáticos y esquistos de biotita- 
muscovita-cuarzo. Los esquistos están cabalgados por las rocas del STT. La edad de estos esquistos no ha sido establecida convincentemente. Algunos autores (de Cserna, 1982; Parga-Perez 1981; Elías-Herrera, 1989) consideraron que son de edad paleozoica, mientras otros los asociaron a la secuencia del arco de Teloloapan y por lo tanto de edad Jurásico Superior-Cretácico Inferior (Talavera-Mendoza 1993).

En la región de Taxco y Taxco el Viejo aflora una secuencia metavolcanoclástica caracterizada por intercalaciones de lavas de composición andesítica y riolítica (De Cserna y Fries 1981; Talavera-Mendoza 1993) con sedimentos volcanoclásticos, tobas, conglomerados volcánicos y niveles de cuarcitas. La edad de esta secuencia está aún sin clarificarse. De Cserna y Fries (1981) propusieron, por correlación con los esquistos del Complejo Acatlán, una edad paleozoica. Igualmente por correlación, se ha asociado con el arco de Teloloapan de edad Mesozoica (Talavera-Mendoza 1993). La secuencia se encuentra cabalgando a las calizas de la Plataforma Guerrero-Morelos.

Estas dos zonas han sido objeto de interpretaciones discrepantes como puede deducirse de los párrafos anteriores. A partir de un análisis estructural y microtectónico, Salinas-Prieto (1994) establece que las rocas de los Esquistos Negros de Tejupilco presentan una historia de deformación más compleja que la registrada en los subterrenos Arcelia y Teloloapan. Los Esquistos Negros de Tejupilco están afectados por tres fases de deformación dúctil de baja temperatura, mientras que la secuencia del STT sólo presenta dos fases sobrepuestas. En la interpretación hecha de estos datos (Salinas-Prieto 1994), se establece que los Esquistos Negros de Tejupilco podrían ser parte del basamento del STT, por lo tanto de edad prejurásica. La estructura y textura presente en los Esquistos Negros de Tejupilco evidencian menor deformación que las rocas del Complejo Acatlán. Por su parte Talavera-Mendoza (1993) propone afinidades entre las rocas del arco de Teloloapan y los Esquistos Negros de Tejupilco, pero diferente de las rocas que aforan en Taxco-Taxco el Viejo.

Con el presente trabajo pretendemos aportar datos que contribuyan al esclarecimiento de la problemática aquí expuesta.

\section{Características del muestreo}

Entre Taxco y Taxco el Viejo, se tomaron 30 muestras de las cuales, sólo la mitad fue estudiada por mictrotermometría de inclusiones fluidas, aunque aquí se consideró toda la columna metamórfica aflorante entre Taxco a Taxco el Viejo. Las muestras corresponden a cuarzo de la primera fase de deformación, relacionada con los esquistos y filitas de la secuencia metamórfica de facies esquistos verdes con protolitos de tobas y lavas (Campa et al. 1974).
En Teloloapan, cuatro muestras (T-01, T-03, T-04 y $\mathrm{T}-05)$ representan al paquete metavolcanoclástico y una (T-02) a la Formación Amatepec. Estas muestras representan los fluidos que participaron en el metamorfismo incipiente de la facies esquistos verdes (Campa et al. 1974). Las deformaciones tectónicas sobreimpuestas no fueron consideradas en este estudio, ya que las inclusiones fluidas secundarias alineadas en microfracturamientos fueron desechadas, y corresponden a efectos sobreimpuestos. Mayores detalles del muestreo y los criterios empleados, así como una descripción de la técnica de preparación y analítica, pueden ser consultados en Moctezuma-Salgado (2000). La localización de los puntos de muestreo se presenta en la Figura 1 y los resultados en la Tabla 1.

\section{Metodología del estudio de las inclusiones fluidas}

\subsection{Consideraciones teóricas}

La transformación de minerales durante el metamorfismo requiere necesariamente de la participación de fluidos calientes. Durante este proceso los cuarzos y calcitas de segregación se han considerado como la fase final del evento, por lo cual las características fisicoquímicas de los fluidos (entre ellas la salinidad, la temperatura y la presión) atrapados en estos minerales nos permiten determinar al menos las condiciones mínimas de presión y temperatura de su formación. La composición de esta solución puede evolucionar en el espacio y en el tiempo. El fenómeno de interacción fluido-roca constituye un fenómeno importante de conocer. Una paragénesis mineral es un conjunto de minerales que cristalizan a un mismo tiempo, es decir, que se encuentran en equilibrio entre ellos y la solución que los originó. La inclusión fluida es una fracción de fluido (líquido y/o vapor) del sistema que quedó atrapada al formarse un cristal (se estima que un $\mathrm{cm}^{3}$ de mineral contiene un millón de inclusiones fluidas). Estas inclusiones guardan una memoria de las condiciones físicas y químicas imperantes en el momento de su formación. Por lo tanto, rastreando la huella de tales fluidos, por medio del estudio de las inclusiones fluidas, se puede reconstruir la historia termobarométrica de una región.

\subsection{Procedimiento práctico}

El análisis se realiza con un microscopio petrográfico de luz transmitida de 1,000 aumentos, al que se le han adicionado una platina térmica que permite el calentamiento de especímenes hasta $\operatorname{los}+650^{\circ} \mathrm{C}$, y un sistema de enfriamiento a base de nitrógeno líquido que puede bajar la temperatura hasta $-200^{\circ} \mathrm{C}$.

El primer paso consiste en reconocer los diferentes tipos de inclusiones y hacer una descripción detallada de 
las mismas, incluyendo su forma, tamaño y fases presentes, que pueden ser sólidas, líquidas o gaseosas. El tamaño pequeño de las inclusiones fluidas, que varía de 5 a 30 micras, hace que esta técnica sea difícil requiriendo de herramientas especializadas para su operación.

La etapa más importante tiene lugar en la microtermometría, técnica que consiste en realizar medidas a bajas y altas temperaturas. El método consiste en provocar la solidificación (bajo observación microscópica) de las fases fluidas, mediante la disminución progresiva de la temperatura (hasta $-200^{\circ} \mathrm{C}$ ). El punto de solidificación de una sustancia es función de su conte-nido salino, por lo cual esta determinación permite el cálculo del porcentaje de sales disueltas en la fase acuosa.

El análisis a altas temperaturas se fundamenta en homogeneizar las diferentes fases presentes a medida que se eleva la temperatura, así como obtener información sobre la temperatura de decrepitación (explosión) de la inclusión. El proceso de calentamiento se efectúa exactamente sobre las mismas inclusiones que se analizaron a baja temperatura, y es indispensable este análisis antes de calentarlas, ya que existe la posibilidad de que se produzca una decrepitación, sobre todo cuando los fluidos son muy densos. La temperatura de homogeneización brinda información sobre la temperatura de formación del cristal.

Los procesos de enfriamiento y calentamiento de las muestras se realizan a una velocidad lenta $\left(1^{\circ} \mathrm{C}\right.$ por minuto) a fin de permitir el equilibrio de las fases y detectar ópticamente los fenómenos de fusión y homogeneización. El amplio rango de temperaturas de observación $\left(-200{ }^{\circ} \mathrm{C}\right.$ a $\left.+650^{\circ} \mathrm{C}\right)$ hacen del método un proceso meticuloso y dispendioso.

\section{Presentación de resultados}

\subsection{Tipos de inclusiones fluidas}

En Tierra Caliente cada unidad litológica presenta características muy distintas en cuanto al contenido de fluidos y su forma. La Figura 2 muestra la morfología de las tres unidades litológicas consideradas en este trabajo y el tipo de fluidos que las caracterizan. La unidad inferior y más antigua, analizada anteriormente por González-Partida (1993b) en los Esquistos Negros de Tejupilco, presenta una esquistosidad de flujo acom-

Tabla 1. Resultados microtermométricos a partir del análisis de las inclusiones fluidas en cuarzo y calcita de segregación.

\begin{tabular}{|c|c|c|c|c|c|}
\hline Muestra & $\begin{array}{c}\text { Th Rango } \\
\left({ }^{\circ} \mathrm{C}\right)\end{array}$ & $\begin{array}{l}\text { Th Promedio } \\
\left({ }^{\circ} \mathbf{C}\right)(\mathbf{n})\end{array}$ & $\begin{array}{l}\text { Tf Rango } \\
\left({ }^{\circ} \mathbf{C}\right)\end{array}$ & $\begin{array}{l}\text { Tf Promedio } \\
\left({ }^{\circ} \mathbf{C}\right)(\mathbf{n})\end{array}$ & $\begin{array}{c}\text { Salinidad Promedio } \\
(\% \text { eq. peso } \mathrm{NaCl})\end{array}$ \\
\hline $\mathrm{T}-01$ & 110 a 149 & $132(10)$ & $+0.7 \mathrm{a}+15.7$ & $+4(13)$ & N.C. \\
\hline $\mathrm{T}-02$ & 133 a158 & $141(5)$ & $-4.9 a+11.3$ & $+2(8)$ & N.C. \\
\hline $\mathrm{T}-03$ & 122 a 188 & 145 (14) & $-3.8 a+21.6$ & $+11.5(9)$ & N.C. \\
\hline $\mathrm{T}-04$ & 120 a 189 & $155(7)$ & $-1.5 a+9.6$ & $+3(8)$ & N.C. \\
\hline T-05 & 126 a 245 & $192(12)$ & -6.4 a -1.5 & $-3(6)$ & 4.9 \\
\hline TX-9701 & 150 a 225 & $206(9)$ & -24.5 a -6.7 & $-15(9)$ & 18.5 \\
\hline TX-9702 & 188 a 257 & $216(9)$ & -84.7 a -77.3 & $-74(6)$ & N.C. \\
\hline TX-9703 & 195 a 296 & $248(9)$ & $-19.3 \mathrm{a}-4.8$ & $-10.6(6)$ & 14.7 \\
\hline M-13 & 199 a 218 & $212(8)$ & - & - & - \\
\hline M-11 & 191 a 232 & 203 (11) & - & - & - \\
\hline M-8 & 198 a 234 & 213 (13) & -32.2 a -2.1 & $-14.9(13)$ & 18.5 \\
\hline TV-9704 & 205 a 233 & $215(10)$ & -18.8 a -4.9 & $-12.3(6)$ & 16.2 \\
\hline TV-9703 & 204 a 221 & $216(8)$ & -19.5 a -20.1 & $-19.8(8)$ & 22.2 \\
\hline M-6 & 213 a 238 & 224 (11) & -10.4 a -2.1 & $-5(10)$ & 7.9 \\
\hline TV-9707 & 213 a 254 & $226(8)$ & -13.4 a -5 & $-7.6(8)$ & 11.2 \\
\hline M-10 & 206 a 258 & 229 (12) & -14.1 a -0.5 & $-6.3(10)$ & 9.6 \\
\hline TV-9706 & 203 a 255 & 237 (16) & -71.7 a -45.9 & $-58.7(5)$ & N.C. \\
\hline
\end{tabular}

N.C.: no calculada; Th: Temperatura de homogenización; Tf: Temperatura de fusión; n: número de inclusiones analizadas; T-02 corresponde a la Formación Amatepec, T-01, T-03, T-04 y T-05 a los metavolcanoclásticos, de Teloloapan, TX-9701, TX-9702, TX-9703, M-6 Y M-IO a los Esquistos Taxco en Taxco, y M-8, M-11, M-13, TV-9703, TV-9704, TV-9706, TV-9707 a los Esquistos en Taxco el viejo. Las muestras de la Formación Amatepec y de los metavolcanoclásticos fueron tomados en las proximidades de Teloloapan. 
pañada de la formación de kink-bands (Figura 2, microfotografías 5, 6 y 7). En los cuarzos de segregación se atraparon fluidos carbónicos del tipo líquido+vapor con la presencia de $\mathrm{CO}_{2}+\mathrm{CH}_{4}+\mathrm{N}_{2}$. En los metavolcanoclásticos y rocas asociadas de las unidades litológicas de Taxco (Tx) y Taxco el Viejo (TV) se presentan inclusiones fluidas del tipo líquido+vapor (Figura 2, fotografías 3 y 4) con relaciones volumétricas del $30-40 \%$ de vapor, considerándose de líquido dominante, y con contenidos de fluidos carbónicos, pero en menor proporción que los Esquistos Negros de Tejupilco, en donde éste es el fluido dominante. En la Formación Amatepec (Figura 2, fotografías 1 y 2) los fluidos son del tipo líquido+vapor (1 a $5 \%$ de vapor) y líquido $100 \%$.

\subsection{Resultados microtermométricos}

\subsubsection{Taxco-Taxco el Viejo}

A baja temperatura los fluidos son de dos tipos: 1) del sistema $\mathrm{H}_{2} \mathrm{O}-\mathrm{NaCl}$ y 2) fluidos con $\mathrm{H}_{2} \mathrm{O}+$ sales+ compuestos carbónicos. Los del primer tipo presentan temperaturas de fusión de $\mathrm{Tf}=-7.6$ a $-19.8^{\circ} \mathrm{C}$, lo que implica salinidades del orden de 11.22 a $22.24 \%$ eq. pe-

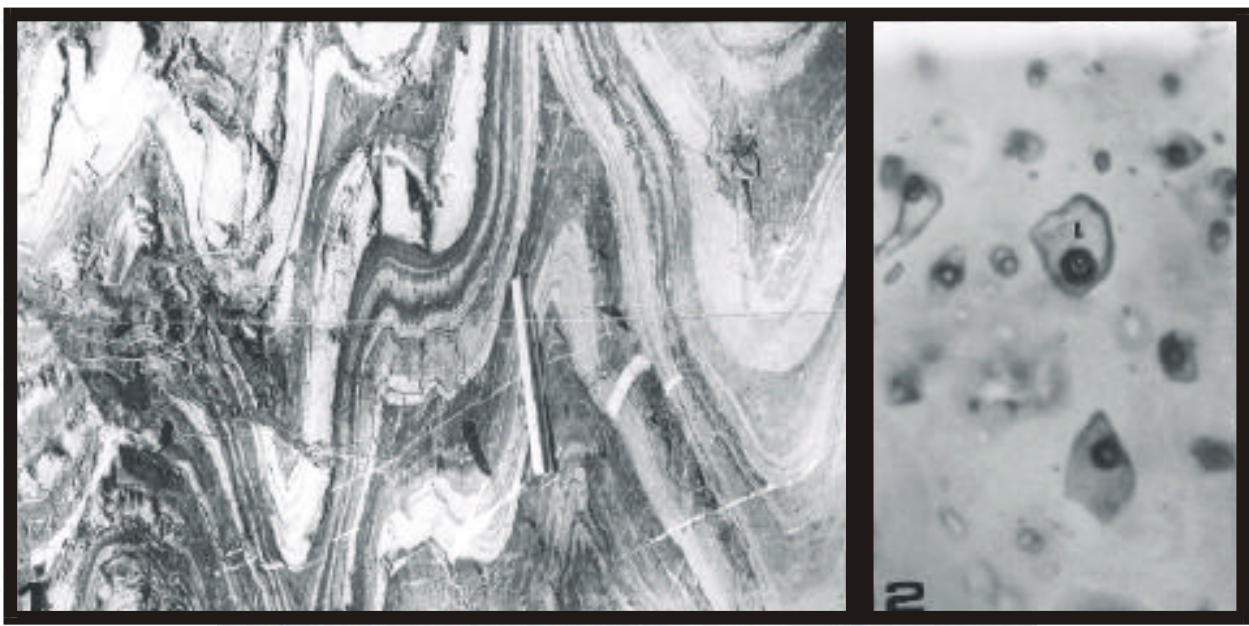

Formación Amatepec. Localidad: Santa Rosa, Edo. de México

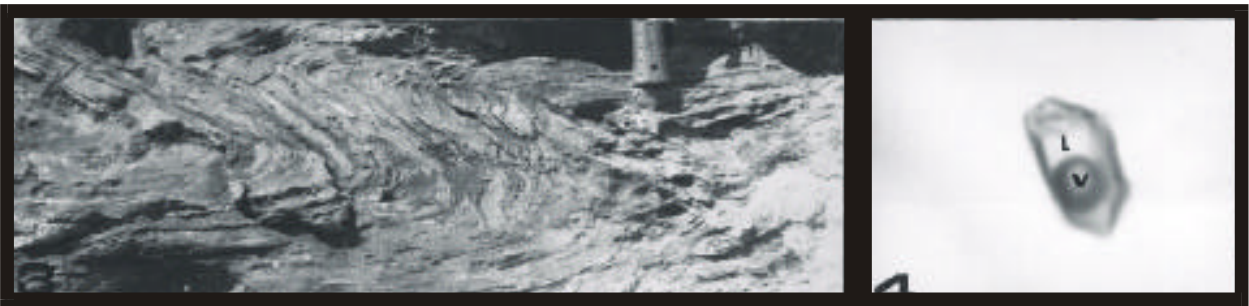

Esquisto Taxco. Localidad: Taxco, Edo. de Guerrero

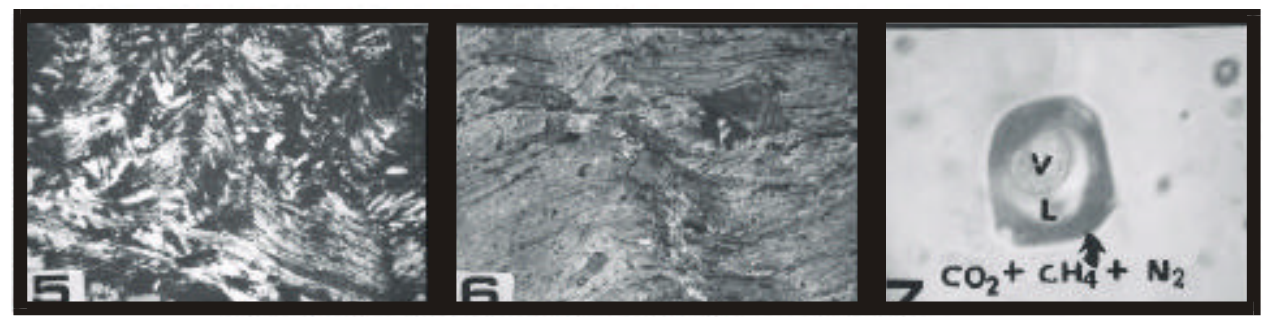

Esquisto Tejupilco. Localidad: Tizapa, Edo. de México

Figura 2. Placas fotográficas de la litología e inclusiones fluidas de: 1) Formación Amatepec (área de Santa Rosa, Edo. de México), pliegues curviplanares. 2) Inclusiones fluidas en calcitas de segregación de la Formación Amatepec, donde L= fase líquida, V= fase vapor. Tamaño de las inclusiones 10 micras. 3) Pliegues isoclinales del esquisto Taxco. 4) Inclusión fluida (15 micras) de los cuarzos de segregación de los esquistos de Taxco, donde $\mathrm{L}=$ líquido y $\mathrm{V}=$ vapor . 5) y 6) Fotomicrografías LP 10x45 de los esquistos negros de Tejupilco. 7) Inclusión fluida carbónica a $\mathrm{CO}_{2}+\mathrm{CH}_{4}+\mathrm{N}_{2}$, donde $\mathrm{L}=$ líquido y V= vapor; tamaño de la inclusión: 20 micras. 
so $\mathrm{NaCl}$. El punto triple del $\mathrm{CO}_{2}$ puro se encuentra a $\mathrm{T}=-56.6^{\circ} \mathrm{C}$, y valores más bajos registrados implican la presencia de otros componentes volátiles como el $\mathrm{CH}_{4} \mathrm{y}$ $\mathrm{N}_{2}$. Este comportamiento lo presentaron el segundo tipo de inclusiones fluidas de los esquistos de Taxco, con $\mathrm{Tf}=-57.8 \mathrm{a}-84.7^{\circ} \mathrm{C}$ y con temperaturas de homogeneización del orden de $\mathrm{Th}=227$ a $296^{\circ} \mathrm{C}$. En los miembros metavolcanoclásticos (muestras $\mathrm{M}$ en la Tabla 1), se presentan temperaturas de fusión de dos tipos: el primero con $\mathrm{Tf}=-2.1 \mathrm{a}-14.1{ }^{\circ} \mathrm{C}$, equivalente a salini-dades de $\mathrm{S}$ $=3.55$ a $17.8 \%$ eq. peso $\mathrm{NaCl}$. Este compor-tamiento es el más general, sin embargo la muestra M-8, presentó un segundo tipo, con $\mathrm{Tf}=-24$ a $-32.2^{\circ} \mathrm{C}$ lo que implica la presencia de $\mathrm{CaCl}$ como salmuera dominante. En este paquete las $\mathrm{Th}=203$ a $248^{\circ} \mathrm{C}$.

\subsubsection{Metavolcanoclásticos de Teloloapan}

Las cuatro muestras analizadas, presentan $\mathrm{Tf}=-1.5^{\circ}$ a $-6.4^{\circ} \mathrm{C}$ lo que corresponde a salinidades de 2.5 a $9.7 \%$ eq. peso $\mathrm{NaCl}$. Algunas inclusiones medidas presentan Tf positivas de $+0.7^{\circ} \mathrm{a}+21.6^{\circ} \mathrm{C}$, lo que implica la fusión de clataratos (mezcla de salmuera con $\mathrm{CO}_{2}$ ), o también muy posiblemente un comportamiento metaestable de los fluidos, por ser éstos los más superficiales. Esta interpretación es apoyada por el hecho de que en estas muestras se observaron inclusiones unifásicas a líquido coexistiendo con las inclusiones fluidas a líquido+vapor. Las temperaturas de homogenei-zación presentan un rango de $\mathrm{Th}=141^{\circ}$ a $192^{\circ} \mathrm{C}$.

\subsubsection{Formación Amatepec}

Las inclusiones fluidas presentan valores de $\mathrm{Th}=110^{\circ}$ a $149^{\circ} \mathrm{C}$, con un promedio de $132^{\circ} \mathrm{C}$. Las temperaturas de fusión son positivas, de $\mathrm{Tf}=+0.7$ a $+15.7^{\circ} \mathrm{C}$, lo que implica la presencia de clataratos, o un comportamiento metaestable, por lo cual no se pudo determinar la salinidad de la fase líquida.

\section{Implicaciones geológicas}

Trabajos de inclusiones fluidas en México han mostrado que los fluidos tienen una importancia considerable en la estabilización del equilibrio fisicoquímico en los terrenos metamórficos y diagenéticos (González-Partida 1993a; Martínez-Ibarra et al., 1994). En Tierra Caliente, los Esquistos Negros de Tejupilco, en el área de Tizapa, Edo. de México, presentan a la base cuarzo+biotita+muscovita+ microclina+albita, acompañados por leucoxenos y turmalina detrítica. La biotita está parcialmente cloritizada, perteneciendo a la facies de esquistos verdes, subfacies de biotita, clase cuarzo-feldespática, aunque Tolson (1993) consideró que puede llegar a la facies de anfibolita. Generalmente el paquete de esquistos se caracteriza por la asociación cuarzo+biotita+muscovita. Con base a datos petrográficos, geoquímicos, cristaloquímicos y de inclusiones fluidas, González-Partida (1993a) determinó que los Esquistos Negros fueron inicialmente arcillas del tipo ilita, formadas a partir de una sedimentación madura, y que su transformación a dos micas (biotita+muscovita) definen un metamorfismo epizonal. A partir de los estudios cristaloquímicos y de inclusiones fluidas, las condiciones termobarométricas de cristalización de las muscovitas varían entre 450$470^{\circ} \mathrm{C}$ y $2.5-4 \mathrm{~Kb}$. Las temperaturas de homogeneización final en las inclusiones fluidas fueron de 300 a $460^{\circ} \mathrm{C}$ con salinidades de $\mathrm{S}=2$ a $8 \%$ eq. peso de $\mathrm{NaCl}$. Los fluidos carbónicos complejos determinados $\left(\mathrm{CO}_{2}+\mathrm{CH}_{4}+\mathrm{N}_{2}\right)$ fueron originalmente materia orgánica dispersa en la fracción arcillosa. En Taxco y Taxco el Viejo la apariencia en campo de las filitas es muy similar a la de los Esquistos Negros de Tejupilco, pero las temperaturas de formación de los cuarzos de segregación en Taxco están en un rango de $\mathrm{Th}=203$ a $248^{\circ} \mathrm{C}$, valor muy inferior al determinado en Tizapa para los Esquistos Negros de Tejupilco. Los metavolcanoclásticos en el área de Teloloapan acusan un rango térmico de la base a la cima de 192 a $141^{\circ} \mathrm{C}$, con fluidos muy distintos a los determinados en las unidades anteriores que presentan complejos fluidos carbónicos. En la Formación Amatepec las temperaturas de homogeneización varían entre 110 y $149^{\circ} \mathrm{C}$.

El comportamiento térmico revelado en el análisis de las inclusiones fluidas es compatible con las observaciones petrológicas, ya que la paragénesis metamórfica de los Esquistos Taxco y rocas metavolcanoclásticos asociadas (Salinas-Prieto 1994) sugiere una historia térmica simple. Por otro lado, la paragénesis metamórfica de las lavas de Arcelia determinada por Talavera-Mendoza (1993) es: prehenita+pumpellita \pm epidota \pm clorita \pm albita y prehenita 土epidota \pm clorita \pm albita. Estas asociaciones pertenecen a las facies prehenita-pumpellita. Talavera-Mendoza (1993) interpreta que existen dos episodios de metamorfismo hidrotermal estático a temperaturas inferiores a los $320^{\circ} \mathrm{C}$ y una presión de $3 \mathrm{~kb}$.

La mayoría de los autores agrupan los Esquistos $\mathrm{Ne}$ gros de Tejupilco con las rocas metavolcanoclásticas de Teloloapan y los esquistos de Taxco y Taxco el Viejo. Esta consideración ha conducido a atribuir edades muy diferentes a todo este conjunto. En efecto, Fries (1960), de Cserna et al. (1974), de Cserna (1982), Parga-Pérez (1981) y Elías-Herrera (1989) han sugerido una edad paleozoica para las formaciones esquistosas y, por otro lado Campa (1978) ha propuesto una edad del Jurásico Superior-Cretácico Inferior. Elías-Herrera y OrtegaGutiérrez (1997) encontraron xenolitos precámbricos en los piroclásticos de la Sierra de la Goleta, lo que implica un basamento viejo para las rocas metavolcanoclásticas. Por otro lado, Salinas-Prieto (1994) define que los Esquistos Negros de Tejupilco son más antiguos y diferentes con respecto de su comportamiento estructural a las rocas metavolcanoclásticas con intercalaciones filíticas del llamado subterreno Teloloapan, en donde las 
rocas de Taxco y Taxco el Viejo forman parte del mismo conjunto petrotectónico de edad Jurásico SuperiorCretácico Inferior. Con base en los estudios del presente trabajo y los resultados publicados con anterioridad por González-Partida (1993a), se sustenta la hipótesis planteada por Salinas-Prieto (1994), ya que entre los Esquistos Negros de Tejupilco y el metavolcanoclástico existen diferencias termobarométricas que no pueden ser explicadas como una evolución gradual, lo que si sucede con el metavolcanoclástico de Teloloapan y los esquistos de Taxco y Taxco el viejo y sus metavolcanoclásticos asociados, en donde las temperaturas van de $\mathrm{Th}=248 \mathrm{a}$ $203^{\circ} \mathrm{C}$ pasando a $\mathrm{Th}=192$ a $141^{\circ} \mathrm{C}$, en este sentido las rocas de Taxco y Taxco el Viejo estarían conformando la parte inferior de la secuencia metavolcanoclástica de Teloloapan, localidad en donde la Formación Amatepec sobreyace a esta unidad presentando bajas temperaturas de homogeneización $\left(\mathrm{Th}=110^{\circ}\right.$ a $\left.149^{\circ} \mathrm{C}\right)$.

\section{Conclusiones}

Los Esquistos Negros de Tejupilco presentan una historia estructural y térmica diferente al paquete metavolcanoclástico aflorante en Taxco y Taxco el Viejo, y en Teloloapan.

Los metavolcanoclásticos de Teloloapan (y esquistos asociados), son parte del mismo conjunto litológico que aflora en Taxco y Taxco el Viejo ya que presentan una evolución térmica gradual.

\section{Agradecimientos}

Agradezco a los editores de la revista, en particular a la Dra. Susana Alaniz Alvarez, a los dos revisores anónimos del manuscrito y a Barbara Martiny por sus comentarios y revisión que contribuyeron al mejoramiento de este trabajo, a Gregorio Solorio M., Crescencio Garduño Paz y Sara Solis en la preparación de muestras, figuras y tabla de resultados. El presente trabajo fue parcialmente financiado por DGAPA-PAPIIT, proyecto IN-100900.

\section{Referencias bibliográficas}

Campa, M. F., 1978, La evolución tectónica de Tierra Caliente, Guerrero: Sociedad Geológica Méxicana, Boletín, 39, 52-64.

Campa, M. F., Campos, R., Flores, R., Oviedo, R., 1974, La secuencia mesozoica volcanosedimentaria metamorfizada de Ixtapan de la Sal México-Teloloapan Guerrero: Boletín de la Sociedad Geológica Mexicana, 35, 7-28.

Campa, M. F., Coney, P. J., 1983, Tectono-stratigraphic Terranes and mineral resource distribution in Mexico: Canadian Journal of Earth Sciences, 20, 1,040-1,051.

Colorado-Lievano, D., 1979, Etude de une partie de la ceinture métamorphique (Mésozoique) du Sud du Mexique (Etats de México et Guerrero): Paris, France, Université Pierre et Marie Curie, Tesis doctoral, $130 \mathrm{p}$.

De Cserna, Z., 1982 (1983), Hoja Tejupilco 14 Q-g (9), Geología de los Estados de Guerrero, México y Michoacán: México, D.F.,
Universidad Nacional Autónoma de México, Instituto de Geología, Carta Geológica de México Serie 1: 100,000, 1 mapa con texto, 28 p.

De Cserna, Z., Fries, C., Rincón-Orta, C., Westley, H., SolorioMunguia, J.G,. Schmitter-Villada, E., 1974, Edad precámbrica tardía del Esquisto Taxco, Estado de Guerrero: Asociación Mexicana de Geólogos Petroleros, Boletín, 26, 183-193.

De Cserna, Z., Fries, C., 1981, Hoja Taxco 14Q-h (7), Geología de los Estados de Guerrero, México y Morelos: México, D.F., Universidad Nacional Autónoma de México, Instituto de Geología, Carta Geológica de México Serie 1: 100.000, 1 mapa con texto, 47 p.

Delgado-Argote, L., López-Martínez, M., York, D., Hall, C. M., 1990, Geocronología del tronco ultramáfico de San Pedro Limón, Edo. de México, y sus implicaciones tectónicas en el complejo Tierra Caliente, in X Convención Geológica Nacional, Libro de resúmenes: México, D.F., Sociedad Geológica Mexicana, 91 (resumen).

Elías-Herrera, M., 1989, Geología metamórfica del área de San Lucas del Maíz, Estado de México: México, D.F., Universidad Nacional Autónoma de México, Instituto de Geología, Boletín, núm. 105, 79 p., 1 mapa.

Elías-Herrera, M., Sánchez-Zavala, J. L., 1990 (1992), Tectonic implications of a mylonitic granite in the lower structural level of the Tierra Caliente Complex (Guerrero Terrane), southern Mexico: México, D.F., Universidad Nacional Autónoma de México, Instituto de Geología, Revista, 9, 113-125.

Elías-Herrera, M., Ortega-Gutiérrez, F., 1997, Petrology of high-grade metapelitic xenoliths in an Oligocene rhyodacite plugPrecambrian crust beneath the southern Guerrero terrane, Mexico?: Revista Mexicana de Ciencias Geológicas, 14, 101-109.

Fries, C., 1960, Geología del Estado de Morelos y partes adyacentes de México y Guerrero, región central meridional de México: México, D.F., Universidad Nacional Autónoma de México, Instituto de Geología, Boletín, núm. 60, 236 p.

González-Partida, E., 1993a, Petrografía, geoquímica, cristaloquímica y características de los fluidos asociados a una sección del metamórfico aflorante en Tierra Caliente, estados de México, Guerrero y Michoacán: Geofísica Internacional, 32, 429-440.

González-Partida, E., 1993b, Datos geoquímicos de roca total para la secuencia volcanosedimentaria Cretácica en Cuale-El Rubí, Jalisco y Tierra Caliente, Guerrero, México: Geofísica Internacional, 32, 249-259.

Martínez-Ibarra, R., González-Partida, E., Guzmán-Vega, M., AraujoMendieta, J., 1994, Fluid Inclusions microthermometry and fluorescence UV studies from outcrop samples of the Tepexic and El Abra Formations, Tampico-Misantla Basin Mexico, in 5a. Conferencia Bienal Panamericana en Investigación sobre Inclusiones Fluidas PACROFI V: Cuernavaca, Morelos, Comisión Federal de Electricidad, Gerencia de Proyectos Geotermoeléctricos, Instituto de Investigaciones Eléctricas, p. 56 (resumen).

Moctezuma-Salgado, M., 2000, Aplicación de la técnica de microtermometría en inclusiones fluidas con ejemplos de Taxco, Taxco el Viejo y Teloloapan (Estado de Guerrero, México): Taxco, Guerero, Universidad Autónoma de Guerrero, Escuela Regional de Ciencias de la Tierra, Tesis profesional, $87 \mathrm{p}$.

Monod, O., Busnardo, R., 1992, Une faune albienne dans la couverture carbonatée de le arc volcanique de Teloloapan (Gro. Mex.), in Table Ronde Européen, Paléontologie et stratigraphie de Amerique Latine: Toulouse, France, Universite de Toulouse, p. 37 (resumen).

Ortiz-Hernández, L. E., Yta, M., Talavera-Mendoza, O., Lapierre, H., Monod, O., Tardy, M., 1991, Origene intra-océanique des formations volcano-plutoniques de arc du Jurassique supérieur-Crétacé inférieur du Mexique centro-méridional: Comptes Rendus de 1'Academie des Siences de Paris, II, 321, 399-406.

Ortiz-Hernández, L. E., 1992., Le arc intra-océanique allochtone Jurassique-Crétacé inférieur du domain cordillerain mexicain (Guerrero Terrane), petrographie, géochimie et minéralisations associées des segments de Guanajuato et Palmar Chico Arcelia. Conséquences paléogéographiques pour le Mésozoique du Mexique: Grenoble, France, Universite Joseph Fourier, Tesis doctoral, 
$312 \mathrm{p}$.

Parga-Pérez, J., 1981, Geología del área de Tizapa, municipio de Zacazonapan, México: México, D.F., Universidad Nacional Autónoma de México, Facultad de Ciencias, Tesis de maestría, 235 p.

Salinas-Prieto, J. C., 1994, Etude structurale du sud-ouest Mexicain (Guerrero)-analyse microtectonique des déformations ductiles du Tertiaire inférieur: Orleans, France, Université de Orléans, Tesis doctoral, Mémoire, núm. 16, 228

Talavera-Mendoza, O., Ramírez-Espinoza, J., Lapierre, H., Monod, O., Campa, M., Tardy, M., 1990, The Albo-Aptian volcanosedimentary calc-alkaline arc series of Teloloapan (Mexico)correlations with the contemporaneus Zihuatanejo sequence and geodynamic implications: $12^{\circ}$ Geowissenschaftliches Lateinamerika-Kolloquium, Munich, Alemania, p. 20 (resumen).

Talavera-Mendoza, O., Ramirez-Espinoza, J., Guerrero-Martínez, M., Salinas-Prieto, J.C., Campa, M., 1992, Petrology and geochemi- cal affinities of the Upper Jurassic-Lower Cretaceous island arc sequences of central-southern Mexico: $14^{\circ}$ Reunión de Ciencias de la Tierra, Universidad de Touluse, Toulouse, Francia, p.147 (resumen).

Talavera-Mendoza, O., 1993, Les formations orogéniques Mésozoiques de Guerrero (Mexique méridional). Contribution á la connaisance de la evolution géodynamique des cordilléres mexicaines: Grenoble, France, Universite, Joseph Fourier, Tesis doctoral, $462 \mathrm{p}$.

Talavera-Mendoza, O., Ramírez-Espinosa, J., Guerrero-Suásteguí, M., 1995, Petrology and geochemistry of the Teloloapan subterranea lower Cretaceous evolved intra-oceanic island-arc: Geofísica Internacional, 34, 3-22.

Tolson, G., 1993, Structural geology and tectonic evolution of the Santa Rosa area, SW Mexico State, Mexico: Geofísica Internacional, 32, 397-413. 